\title{
Impact of non uniform strain configuration on transport properties for FD14+ devices
}

\author{
C. Medina-Bailon ${ }^{1,2}$, C. Sampedro ${ }^{1,2}$, F. Gámiz ${ }^{1,2}$, A. Godoy ${ }^{1,2}$, and L. Donetti ${ }^{1,2}$ \\ ${ }^{1}$ Nanoelectronics Research Group. Departamento de Electrónica. \\ Universidad de Granada. Campus Fuentenueva S/N. 18071 Granada. Spain. \\ ${ }^{2}$ CITIC Universidad de Granada. 18071 Granada. Spain. \\ E-mail: csampe@ugr.es
}

\begin{abstract}
As device dimensions are scaled down, the use of non-geometrical performance boosters becomes of special relevance. In this sense, strained channels are proposed for the $14 \mathrm{~nm}$ FDSOI node. However this option may introduce a new source of variability since strain distribution inside the channel is not uniform at such scales. In this work, a MS-EMC study of different strain configurations including non-uniformities is presented showing drain current degradation because of the increase of intervalley phonon scattering and the subsequent variations of transport effective mass and drift velocity. This effect, which has an intrinsic statistical origin, will make necessary further optimizations to keep the expected boosting capabilities of strained channels.
\end{abstract}




\section{INTRODUCTION}

In the seek of improved performance and lower power consumption in MOSFETs, the 28/20nm CMOS technology drives at a milestone as the successful bulk technology became deprecated because of the difficulty of fulfilling the requirements given by the ITRS for the forthcoming technological nodes [1]. In particular, standard bulk-MOSFET technology cannot provide satisfactory solutions for sub-22 nm nodes due to the limited control of SCEs and variability problems arising from a highly doped channel [2]. Different technological choices like multigate architectures [3-5], planar Fully-Depleted Silicon-On-Insulator (FDSOI) [610] or the co-integration of III-V and Ge devices on SOI substrates [11] are competing trying to positioning themselves for the midterm technological scenario. FDSOI devices constitute the natural continuation in planar technology for future nodes thanks to their technological compatibility, outstanding electrostatic control, simpler fabrication process, and competitive overall cost. For the $14 \mathrm{~nm}$ node, two generations have been proposed for commercial applications using FDSOI technology. The first one, FD14, will be implemented considering a gate of $20 \mathrm{~nm}$ with an overlapped doping profile which yields a shorter effective channel length. The use of a silicon slab as thin as $6 \mathrm{~nm}$ and ultrathin buried oxide (UTBOX) with different back bias configurations will ensure a powerful and flexible platform for both high performance (HP) and low power (LP) applications. The evolution of this node, FD14+, will be focused on improved performance rather than in geometrical scaling. In this way, strained channels will be included in order to boost the carrier mobility [12-14]. However, at such dimensions, the high lateral field and confinement conditions may affect in an important manner the expected transport properties of FDSOI transistors. On the other hand, it is very difficult to induce uniform stress profiles as already shown by structural characterization techniques such as dark-field electron holography [15]. This fact may add a new source of variability with impact on the performance of future devices and circuits. Within this framework, advanced device simulation represents an invaluable tool for the assessment of upcoming technological options in two ways: predicting the performance of different architectures and technological choices; and evaluating optimization options in order to reduce the development stage in terms of cost and time. Another important advantage is the possibility of performing thorough studies of the impact of each physical effect and technological booster separately to explain experimental results and to determine which is the dominant 
one on the performance of the considered devices. Therefore, the aim of this work is the study, through Multi-Subband Ensemble Monte Carlo simulations (MS-EMC), of the impact of non-uniform strain distributions in FD14+ technology considering different strain configurations.

\section{SIMULATION METHODOLOGY}

The fundamentals of Multi-Subband (MS) algorithms are based on the mode-space approach of quantum transport [16], which separates the physical description of the simulation domain into a confined and a non-confined problem. In this way, the device is divided in several slices along the confinement direction where the 1D Schrödinger equation is solved. The transport properties are obtained thanks to the solution of the 2D Boltzmann Transport Equation (BTE) in the perpendicular plane (X-Y in Figure 1) considering an ensemble of charged superparticles describing the electrons inside the simulation domain. The semiclassical and quantum descriptions of the device are coupled by means of the $2 \mathrm{D}$ solution of Poisson equation in order to keep the self-consistency of the simulator.

MS-EMC simulators provide one of the most accurate descriptions of carrier transport since detailed scattering models are included while the main part of quantum effects are taken into account keeping an affordable computational cost. This is the reason why, this method has been extensively used by different groups worldwide [17-21]. This code has been calibrated by comparing to experimental data obtained from [22] and with MS deterministic simulators as shown in [23]. In the same way it has demonstrated its capabilities studying different advanced bulk and SOI planar nanodevices [24-27]. Concerning the description of the conduction band, the effective mass approximation is used assuming non-parabolicity corrections. The transport model includes the most important scattering mechanisms i.e. acoustic and intervalley phonon [17], surface roughness [28] and Coulomb interaction. Arbitrary strain conditions can be locally considered tailoring the energy minima of each valley in order to reproduce uniaxial, biaxial, combined (the addition of biaxial and uniaxial) or non-uniform analytical strain mapping.

To perform the aforementioned study to determine the impact of non-uniform strain on ultrascaled FDSOI transistors, a template device with a gate length of $20 \mathrm{~nm}$, a channel 
thickness $\mathrm{T}_{S i}=6 \mathrm{~nm}$ and EOT $=1 \mathrm{~nm}$ has been considered as depicted in Figure 2. Midgap metal gate and $\mathrm{P}+$ back plane with a $10 \mathrm{~nm}$ UTBOX has been also used. The confinement and transport directions were set as $<100>$ and $<110>$ respectively where $\Delta_{2}$ valleys present the smaller conduction mass $\left(m_{t}=0.19 m_{0}\right)$. The doping profile has been chosen to fit the effective channel length specifications for FD14 devices with $\mathrm{N}_{\text {Dpeak }}=$ $1 \times 10^{20} \mathrm{~cm}^{-3}$ and a Gaussian lateral decay which produces an overlapped transition region (Figure 2 symbols). Biaxial (sSOI with a $\mathrm{Si}_{0.8} \mathrm{Ge}_{0.2}$ virtual substrate), uniaxial (1 GPa) and a combination of the two previous strain conditions have been considered, including two cases of non-uniform strain with linear decay for each configuration. These distributions have been chosen after the analysis of those obtained from TCAD process simulation following the structures depicted in [29] (dashed lines in Figure 2). According to this, it has been considered that the strength of the strain decays from the S/D contacts to the center of the channel a $50 \%$ and a $100 \%$ respectively, as shown in Figure 2 (right side axis solid line).

\section{RESULTS AND DISCUSSION}

A set of simulations including linear and saturation bias conditions has been performed to determine the importance of non-uniform strain distributions on device performance. $\mathrm{I}_{D}$ vs. $\mathrm{V}_{G S}$ curves with $\mathrm{V}_{D S}=100 \mathrm{mV}$ for the different configurations of biaxial strain (sSOI with $\mathrm{Si}_{0.8} \mathrm{Ge}_{0.2}$ virtual substrate) are shown in Figure 3. Two main effects can be observed in this plot. On the one hand, the increase observed in $\mathrm{I}_{O N}$ level when uniform strain conditions are considered is reduced as non-uniformities appear in the channel ranging from $32 \%$ to $14 \%$ for $V_{G}=V_{D D}$. On the other hand it is also observed a decrease in $\mathrm{V}_{t h}$ for the uniform distribution approaching to the values corresponding to the relaxed device as the non-uniform strain becomes more important. This reduction for strained devices is produced because of the shifting of the energy minima of $\Delta_{2}$ valleys for strained channels, being necessary a smaller gate voltage to reach charge inversion conditions.

The impact of strain non-uniformities as lateral field is increased can be observed in Figure 4. There, current ratios referenced to the non-strained device as a function of $\mathrm{V}_{D S}$ are represented for biaxial (top), uniaxial (middle) and combined (bottom) strain distributions. In all the cases, the enhancement in the drain current as a consequence of 
strain is reduced as $\mathrm{V}_{D S}$ grows showing a plateau when saturation condition is reached. Figure 4 also shows that saturation in current enhancement is achieved for smaller drain voltages as strain distribution varies in a significant way along the channel.

Considering that drain current is proportional to the inversion charge and the drift velocity of the carriers, it is interesting to check which of the two magnitudes is affected the most by strain. Figure 5 demonstrates that there is a small impact of strain distribution on the inversion charge for $\mathrm{V}_{G}>\mathrm{V}_{t h}$ under both linear and saturation transport conditions (top and bottom respectively). Therefore, it is expected that the main impact of strain variations should be observed in velocity profiles.

Figure 6 shows the average velocity as a function of the position for the biaxial case under low (top) and saturation (bottom) drain bias conditions. Due to the ultra scaled device geometry, the maximum value of velocity does not appear in the channel-drain edge but inside the channel. As observed, there is a bigger degradation of velocity profiles as non-uniformities become more important and drain bias is increased, approaching to the values obtained for the relaxed channel. Figure 6 also shows a shifting of velocity maxima from the ending region of the channel to the center as the strain is more uniform. This trend to a symmetrical velocity profile indicates the enhancement of the quasi-ballistic behavior thanks to the strain [27].

There are two main reasons that explain this degradation of transport properties as non-uniform strain distributions are considered. On the one hand, there is an increase of the scattering in the channel region under non-uniform strain conditions as shown in Figure 7 (top). The reduction in the energy shifting among valleys due to strain relaxation increases the scattering rate respect to the uniform strain case with the consequent reduction of the drift velocity. Intervalley phonon scattering is the main responsible of this effect as shown in Figure 7 (bottom) where the differences in the scattering among considered devices basically lays on this mechanism. It is also worth to highlight the importance of scattering in quasi-ballistic transport, small differences in the average number of events per electron (around 0.5 events between relaxed and uniform strained devices) lead to variations in the drain current up to $20 \%$ (Figure 4 top). 
On the other hand, this scattering rate increase (which appears under all drain bias conditions) has a side effect especially in the saturation regime that causes an extra reduction of the drift velocity. The enhancement of intervalley scattering leads to a repopulation of primed subbands $\left(\Delta_{4}\right)$ since electrons are transferred from the non-primed subbands (belonging to $\Delta_{2}$ valleys) to the primed ones as depicted in Figure 8 for biaxial strain conditions at low drain voltage (top) and saturation regime (bottom). This reduction in the population increases the average transport effective mass with the consequent decrease of the drift velocity. The combination of both effects is responsible of the loss of strain efficiency, especially in the saturation region, reducing the benefits of strained channels.

\section{CONCLUSION}

This work presents a MS-EMC study of the impact of non-uniform strain distributions in FD14+ devices. Our simulations show an important degradation of device performance as the strain configuration varies along the channel. This effect becomes of special interest at high drain voltage conditions where the enhancement of intervalley scattering causes a loss of the boosting capabilities of strained channels not only because of the scattering itself but also due to the increase in the transport effective mass as a consequence of the repopulation of primed subbands. This effect, which has an intrinsic statistical origin, may be considered as a new source of device variability in future technological nodes, making necessary further optimizations in this respect.

\section{ACKNOWLEDGMENT}

The authors would like to thanks the support given by Spanish Government (FIS201126005, TEC2011-28660), CEI-BIOTIC mP-TIC-12 and Junta de Andalucia (P10-TIC-6902).

[1] International Technology Roadmap for the Semiconductor Industry, http://www.itrs.net/. 
[2] C. Millar, D. Reid, G. Roy, S. Roy, A. Asenov, Accurate Statistical Description of Random Dopant-Induced Threshold Voltage Variability, Electron Device Letters, IEEE 29 (8) (2008) $946-948$.

[3] F. J. Garcia Ruiz, A. Godoy, F. Gamiz, C. Sampedro, L. Donetti, A comprehensive study of the corner effects in Pi-gate MOSFETs including quantum effects, Electron Devices, IEEE Transactions on 54 (12) (2007) 3369-3377.

[4] J.-P. Colinge, FinFETs and Other Multi-Gate Transistors, Springer Publishing Company, 2007.

[5] K. Kuhn, M. Liu, H. Kennel, Technology options for 22nm and beyond, in: Junction Technology (IWJT), 2010 International Workshop on, 2010, pp. 1 -6.

[6] F. Balestra, J. Brini, P. Gentil, Deep depleted SOI MOSFETs with back potential control: A numerical simulation, Solid-State Electronics 28 (10) (1985) 1031 - 1037.

[7] F. Balestra, S. Cristoloveanu, M. Benachir, J. Brini, T. Elewa, Double-gate Silicon-OnInsulator Transistor with Volume Inversion: A New Device with Greatly Enhanced Performance, IEEE Elec. Dev. Lett. 8 (9) (1987) 410-412.

[8] J. Colinge, Subthreshold slope of thin-film SOI MOSFET's, Electron Device Letters, IEEE 7 (4) (1986) 244-246.

[9] A. Khakifirooz, K. Cheng, B. Jagannathan, P. Kulkarni, J. Sleight, D. Shahrjerdi, J. Chang, S. Lee, J. Li, H. Bu, R. Gauthier, B. Doris, G. Shahidi, Fully depleted extremely thin SOI for mainstream 20nm low-power technology and beyond, in: Solid-State Circuits Conference Digest of Technical Papers (ISSCC), 2010 IEEE International, 2010, pp. $152-153$.

[10] A. Khakifirooz, K. Cheng, A. Reznicek, T. Adam, N. Loubet, H. He, J. Kuss, J. Li, P. Kulkarni, S. Ponoth, R. Sreenivasan, Q. Liu, B. Doris, G. Shahidi, Scalability of Extremely Thin SOI (ETSOI) MOSFETs to Sub-20-nm Gate Length, Electron Device Letters, IEEE 33 (2) (2012) $149-151$.

[11] M. Yokoyama, S. Kim, R. Zhang, N. Taoka, Y. Urabe, T. Maeda, H. Takagi, T. Yasuda, H. Yamada, O. Ichikawa, N. Fukuhara, M. Hata, M. Sugiyama, Y. Nakano, M. Takenaka, S. Takagi, CMOS integration of InGaAs nMOSFETs and Ge pMOSFETs with self-align Nibased metal S/D using direct wafer bonding, in: VLSI Technology (VLSIT), 2011 Symposium on, 2011, pp. $60-61$.

[12] S. Deleonibus, F. Andrieu, P. Batude, X. Jehl, F. Martin, F. Milesi, S. Morvan, F. Nemouchi, 
M. Sanquer, M. Vinet, Future micro/nano-electronics: Towards full 3D and zero variability, in: Junction Technology (IWJT), 2013 13th International Workshop on, 2013, pp. 1-5.

[13] L. Grenouillet, Q. Liu, R. Wacquez, P. Morin, N. Loubet, D. Cooper, A. Pofelski, W. Weng, F. Bauman, M. Gribelyuk, Y. Wang, B. De Salvo, J. Gimbert, K. Cheng, Y. Le Tiec, D. Chanemougame, E. Augendre, S. Maitrejean, A. Khakifirooz, J. Kuss, R. Schulz, C. Janicki, B. Lherron, S. Guillaumet, O. Rozeau, F. Chafik, J. Bataillon, T. Wu, W. Kleemeier, M. Celik, O. Faynot, R. Sampson, B. Doris, M. Vinet, UTBB FDSOI scaling enablers for the 10nm node, in: SOI-3D-Subthreshold Microelectronics Technology Unified Conference (S3S), 2013 IEEE, 2013, pp. 1-2.

[14] O. Faynot, F. Andrieu, C. Fenouillet-Beranger, O. Weber, P. Perreau, L. Tosti, L. Brevard, O. Rozeau, P. Scheiblin, O. Thomas, T. Poiroux, Planar FDSOI technology for sub 22nm nodes, in: VLSI Technology Systems and Applications (VLSI-TSA), 2010 International Symposium on, 2010, pp. 26-27.

[15] D. Cooper, A. Beche, J. M. Hartmann, V. Carron, J.-L. Rouviere, Strain mapping for the semiconductor industry by dark-field electron holography and nanobeam electron diffraction with nm resolution, Semiconductor Science and Technology 25 (9) (2010) 095012.

[16] R. Venugopal, Z. Ren, S. Datta, M. S. Lundstrom, D. Jovanovic, Simulating Quantum Transport in Nanoscale Transistors: Real Versus Mode-Space Approaches, Journal of Applied Physics 92 (2002) 3730-3739. \path\{doi:10.1063/1.1503165\}.

[17] M. V. Fischetti, S. E. Laux, Monte Carlo Study of Electron Transport in Silicon Inversion Layers, Physical Rev. B 48 (4) (1993) 2244-2274.

[18] J. Saint-Martin, A. Bournel, F. Monsef, C. Chassat, P. Dollfus, Multi Sub-Band Monte Carlo Simulation of an Ultra-Thin Double Gate MOSFET with 2D Electron Gas, Sem. Sci. and Tech. 21 (4) (2006) 29-31.

[19] E. Sangiorgi, P. Palestri, D. Esseni, C. Fiegna, L. Selmi, The Monte Carlo approach to transport modeling in deca-nanometer MOSFETs, Solid-State Electronics 52 (9) (2008) 1414 1423.

[20] P. Palestri, C. Alexander, A. Asenov, V. Aubry-Fortuna, G. Baccarani, A. Bournel, M. Braccioli, B. Cheng, P. Dollfus, A. Esposito, D. Esseni, C. Fenouillet-Beranger, C. Fiegna, G. Fiori, A. Ghetti, G. Iannaccone, A. Martinez, B. Majkusiak, S. Monfray, V. Peikert, S. Reggiani, C. Riddet, J. Saint-Martin, E. Sangiorgi, A. Schenk, L. Selmi, L. Silvestri, P. Toniutti, J. Wal- 
czak, A comparison of advanced transport models for the computation of the drain current in nanoscale nMOSFETs, Solid-State Electronics 53 (12) (2009) 1293 - 1302.

[21] C. Sampedro, F. Gámiz, A. Godoy, R. Valín, A. García-Loureiro, F. Ruiz, Multi-Subband Monte Carlo study of device orientation effects in ultra-short channel DGSOI, Solid-State Electronics 54 (2) (2010) $131-136$.

[22] C. Fenouillet-Beranger, P. Perreau, S. Denorme, L. Tosti, F. Andrieu, O. Weber, S. Monfray, S. Barnola, C. Arvet, Y. Campidelli, S. Haendler, R. Beneyton, C. Perrot, C. de Buttet, P. Gros, L. Pham-Nguyen, F. Leverd, P. Gouraud, F. Abbate, F. Baron, A. Torres, C. Laviron, L. Pinzelli, J. Vetier, C. Borowiak, A. Margain, D. Delprat, F. Boedt, K. Bourdelle, B.-Y. Nguyen, O. Faynot, T. Skotnicki, Impact of a $10 \mathrm{~nm}$ ultra-thin BOX (UTBOX) and ground plane on FDSOI devices for $32 \mathrm{~nm}$ node and below, Solid-State Electronics 54 (9) (2010) 849 -854 .

[23] F. Vecil, J. M. Mantas, M. J. Cáceres, C. Sampedro, A. Godoy, F. Gámiz, A parallel deterministic solver for the Schrödinger-Poisson-Boltzmann system in ultra-short DG-MOSFETs: Comparison with Monte-Carlo, Computers \& Mathematics with Applications 67 (9) (2014) $1703-1721$.

[24] C. Sampedro, F. Gámiz, A. Godoy, R. Valín, A. García-Loureiro, N. Rodríguez, I. TiendaLuna, F. Martinez-Carricondo, B. Biel, Multi-Subband Ensemble Monte Carlo simulation of bulk MOSFETs for the 32 nm-node and beyond, Solid-State Electronics 65-66 (2011) 88 - 93.

[25] J. Saint-Martin, A. Bournel, P. Dollfus, Comparison of multiple-gate MOSFET architectures using Monte Carlo simulation, Solid-State Electronics 50 (1) (2006) 94 - 101.

[26] C. Sampedro, F. Gámiz, L. Donetti, A. Godoy, Reaching sub-32 nm nodes: ET-FDSOI and BOX optimization, Solid-State Electronics 70 (2012) $101-105$.

[27] A. Mangla, J.-M. Sallese, C. Sampedro, F. Gamiz, C. Enz, Modeling the Channel Charge and Potential in Quasi-Ballistic Nanoscale Double-Gate MOSFETs, Electron Devices, IEEE Transactions on 61 (8) (2014) 2640-2646.

[28] F. Gámiz, P. Cartujo-Cassinello, J. B. Roldán, F. Jiménez-Molinos, Electron Transport in Dtrained Si Inversion Layers Grown on SiGe-On-Insulator Substrates, Journal of Applied Physics 92 (1) (2002) 288-295.

[29] F. Payet, F. Boeuf, C. Ortolland, T. Skotnicki, Nonuniform Mobility-Enhancement Techniques and their Impact on Device Performance, Electron Devices, IEEE Transactions on 55 (4) 
(2008) 1050-1057. 
Figures 


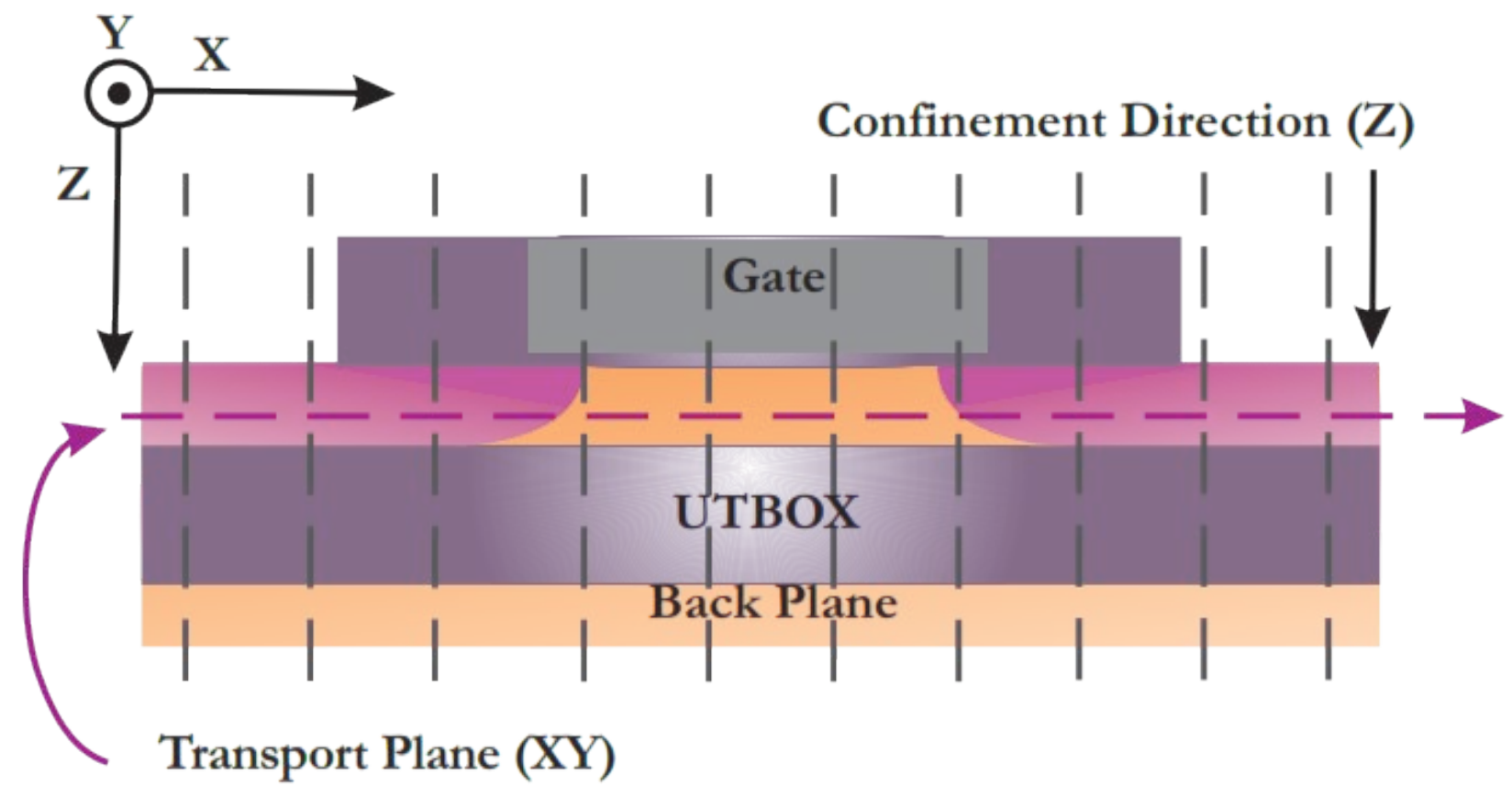

Figure 1: FDSOI with Back-Plane structure for MS-EMC simulation. 


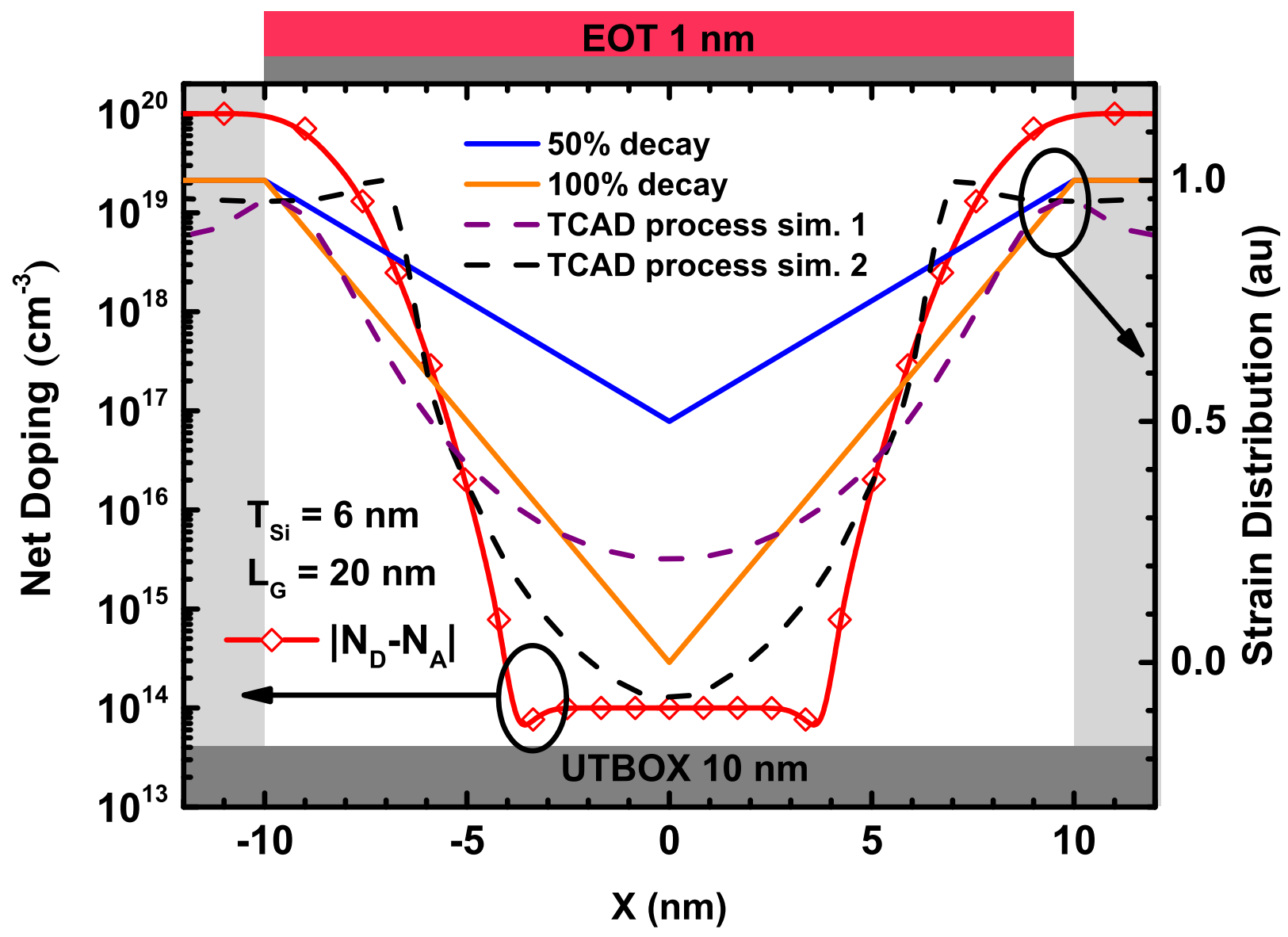

Figure 2: Simulated FDSOI structure including net doping profile (left axis, solid line with symbols). Different strain distributions are shown in right axis including analytical profiles used in this work (solid) and values extracted from TCAD process simulations (dashed). 


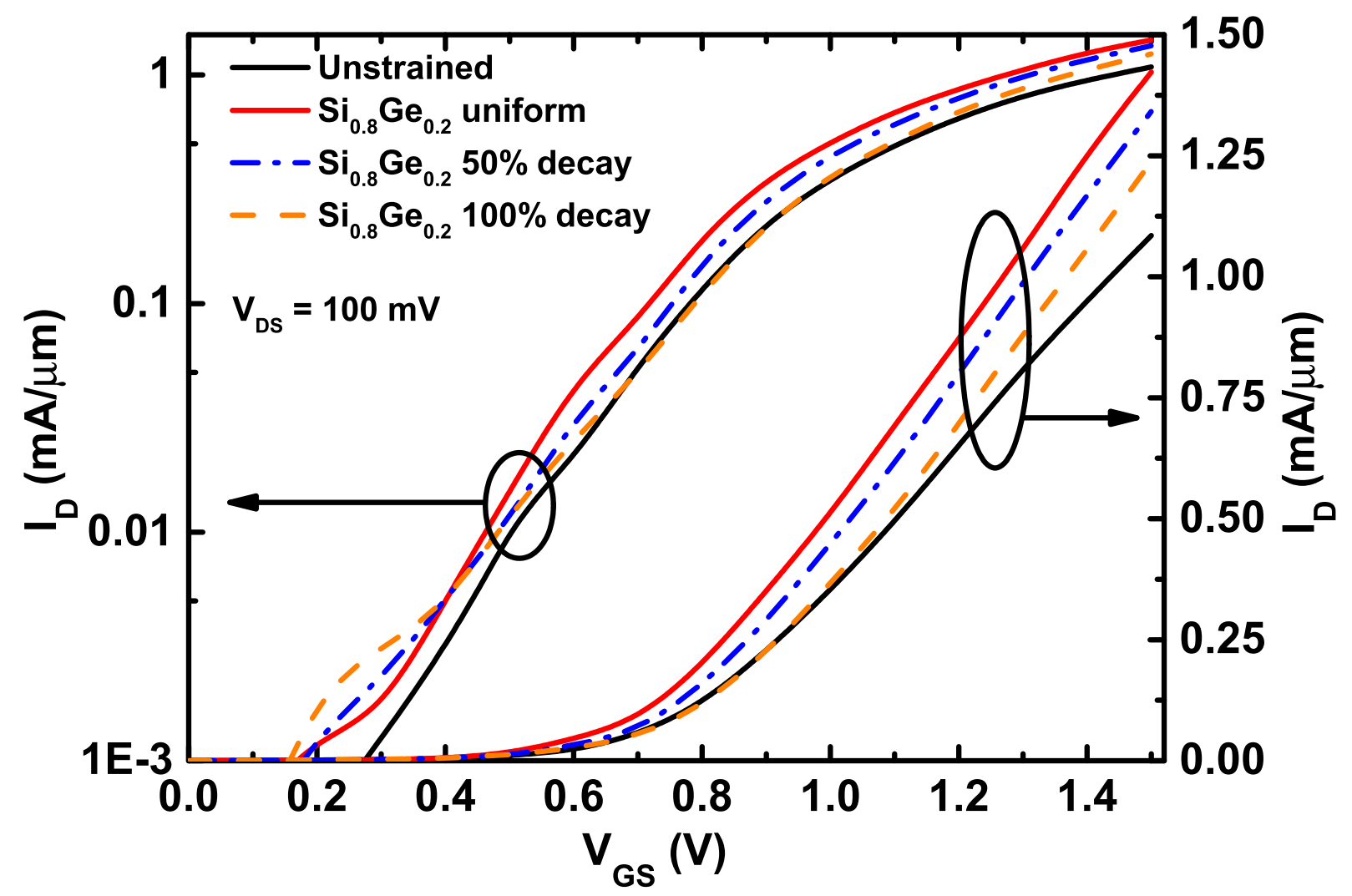

Figure 3: $\mathrm{I}_{D}$ vs. $\mathrm{V}_{G S}$ under low drain bias condition for sSOI devices considering different strain configurations. For the sake of clarity log and linear scales are shown on the left and right axes respectively 

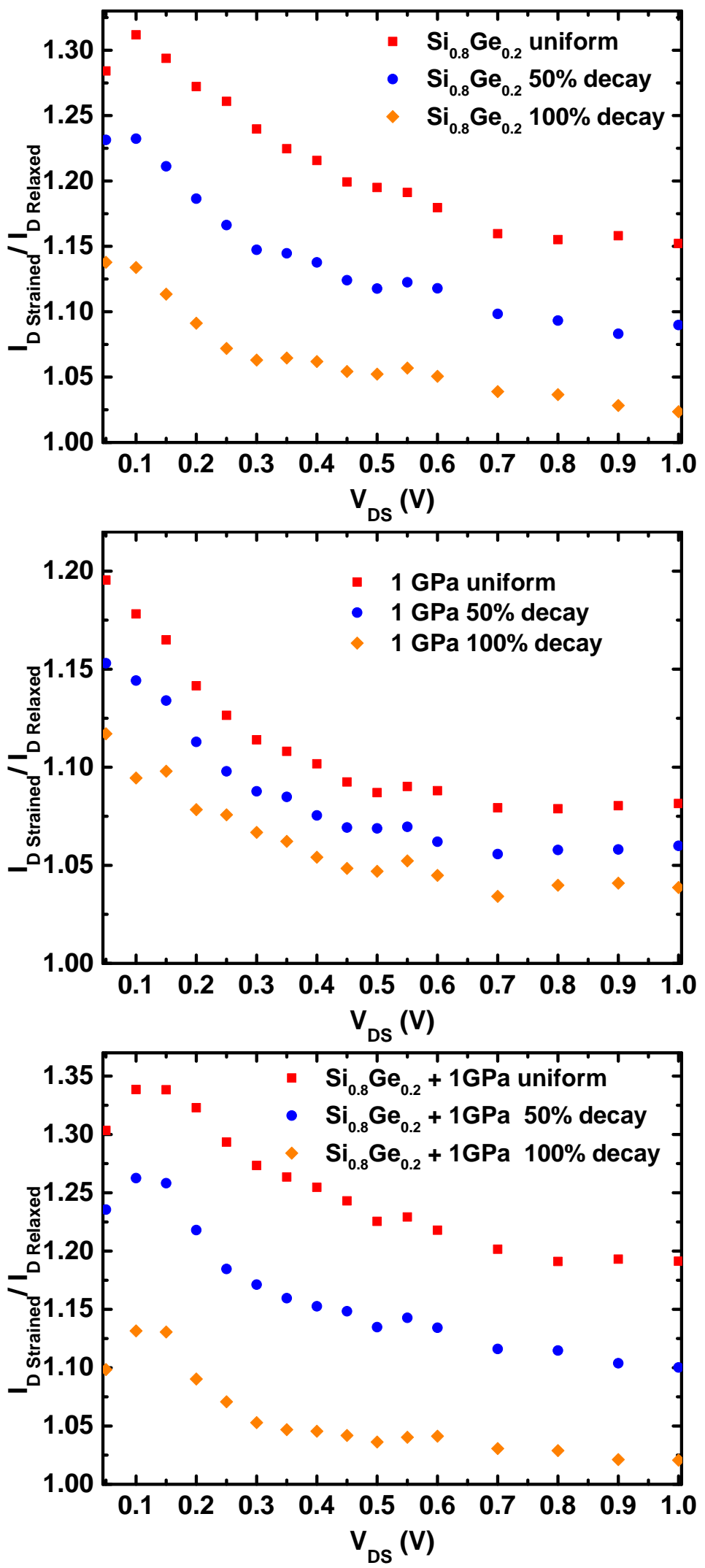

Figure 4: Drain current ratio referenced to the non-strained device as a function of $\mathrm{V}_{D S}$ for biaxial (top), uniaxial (middle) and combined (bottom) strain distributions at $\mathrm{V}_{G S}=1 \mathrm{~V}$. 

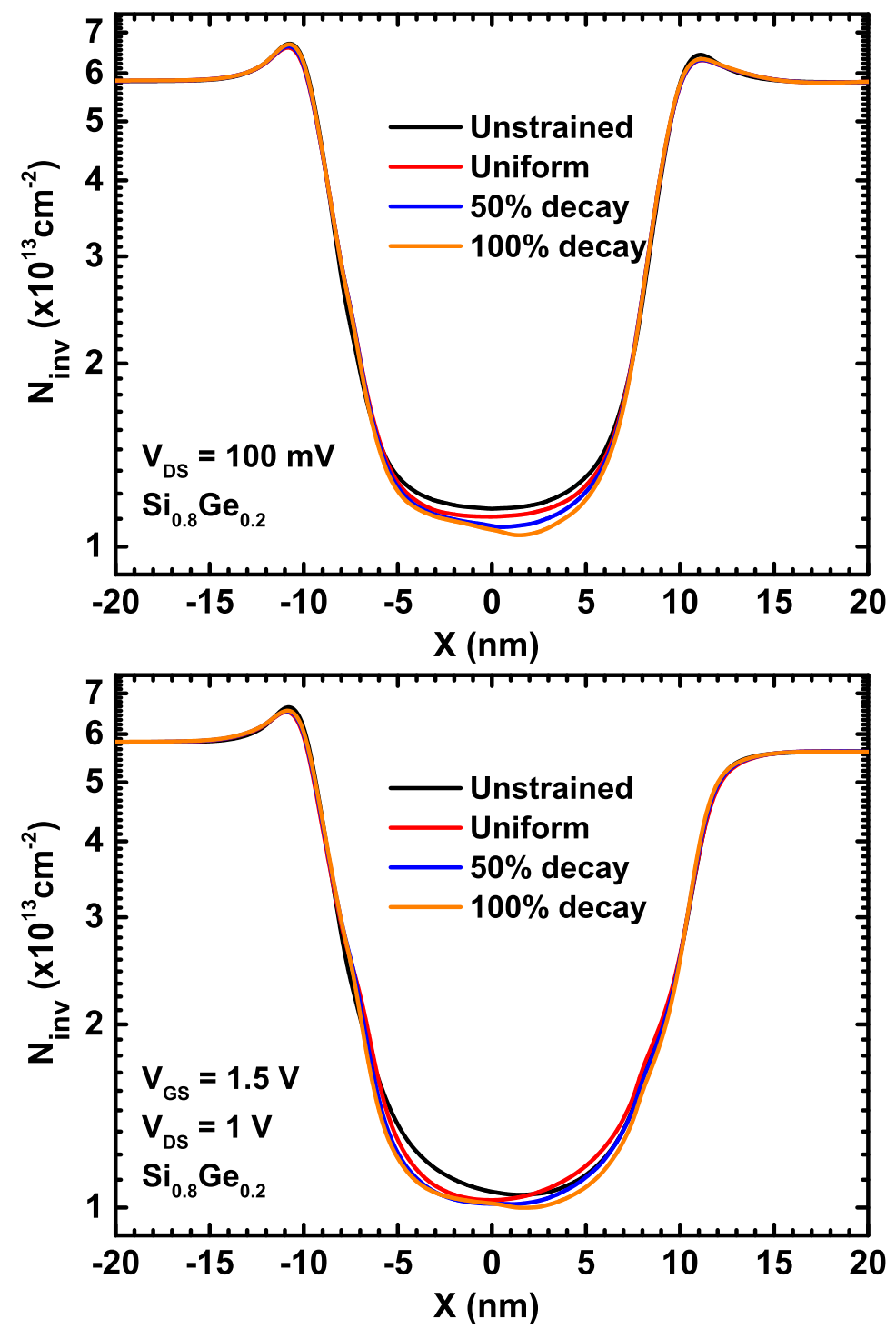

Figure 5: Inversion charge plot for different strain distributions of sSOI devices under linear (top) and saturation (bottom) conditions. 

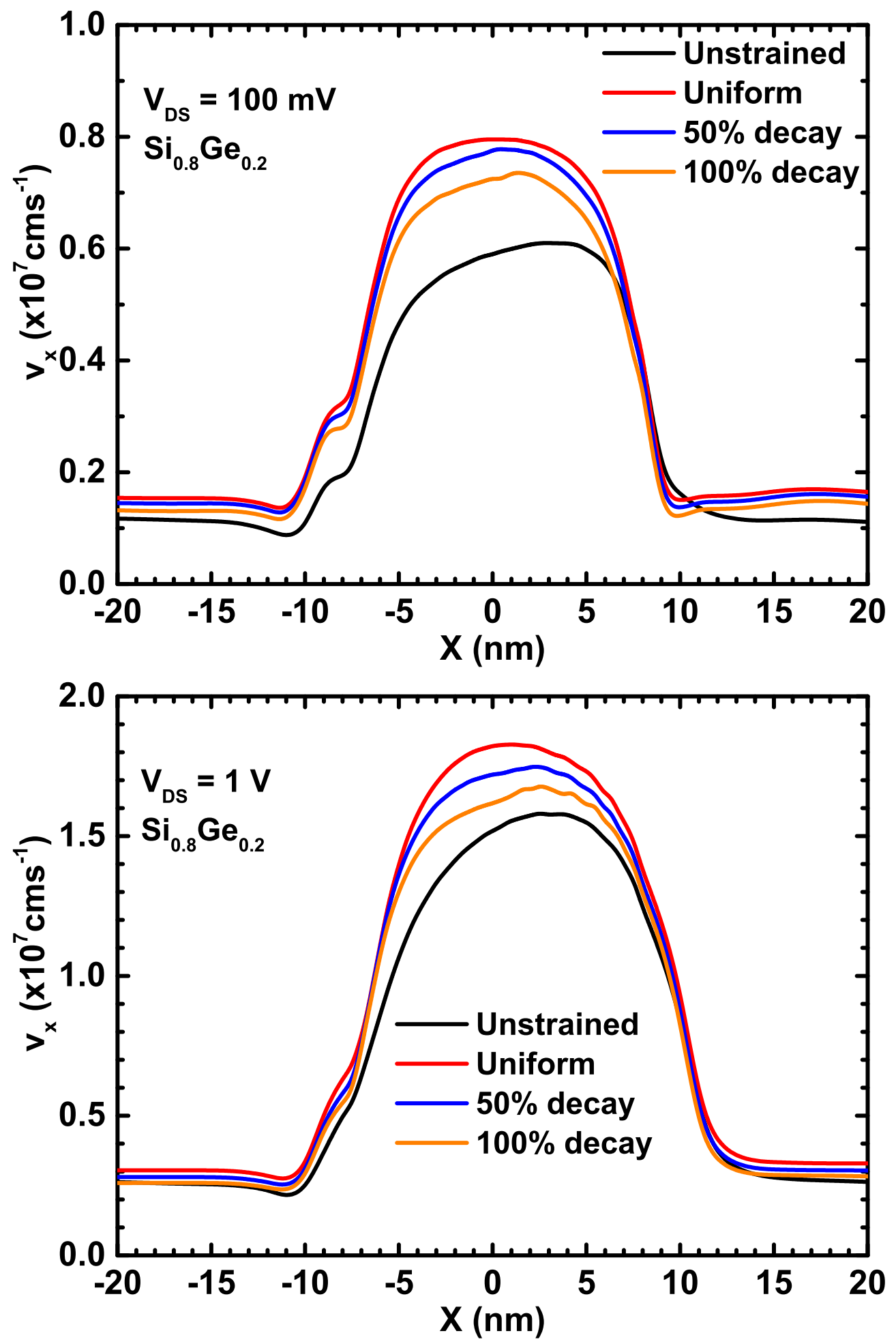

Figure 6: Drift velocity profile for sSOI devices under low drain bias (top) and saturation (bottom) conditions. 

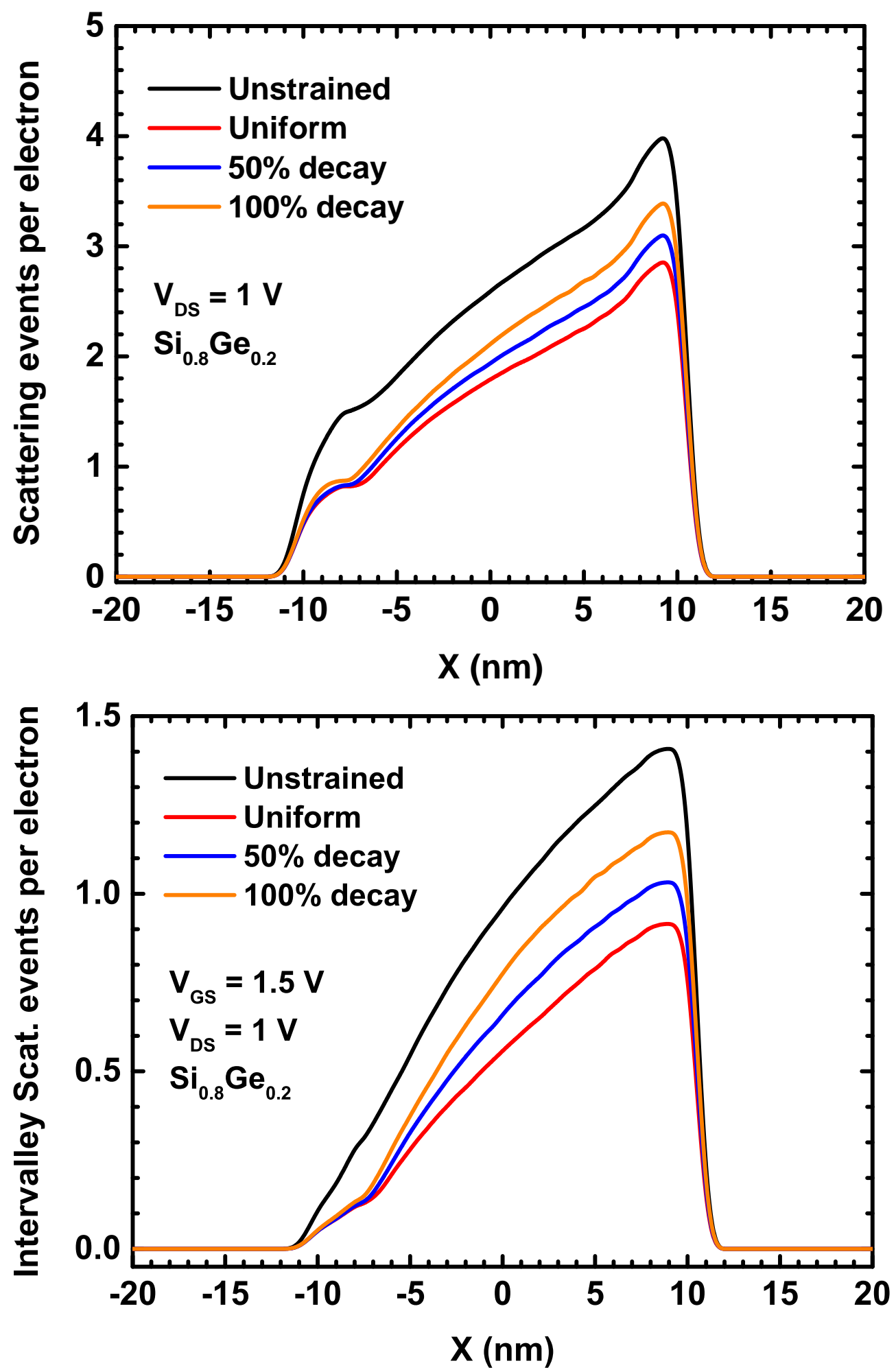

Figure 7: Accumulated number of scattering events per electron in the gated region for sSOI devices as a function of the position (top). Contribution of intervalley phonon scattering (bottom). 

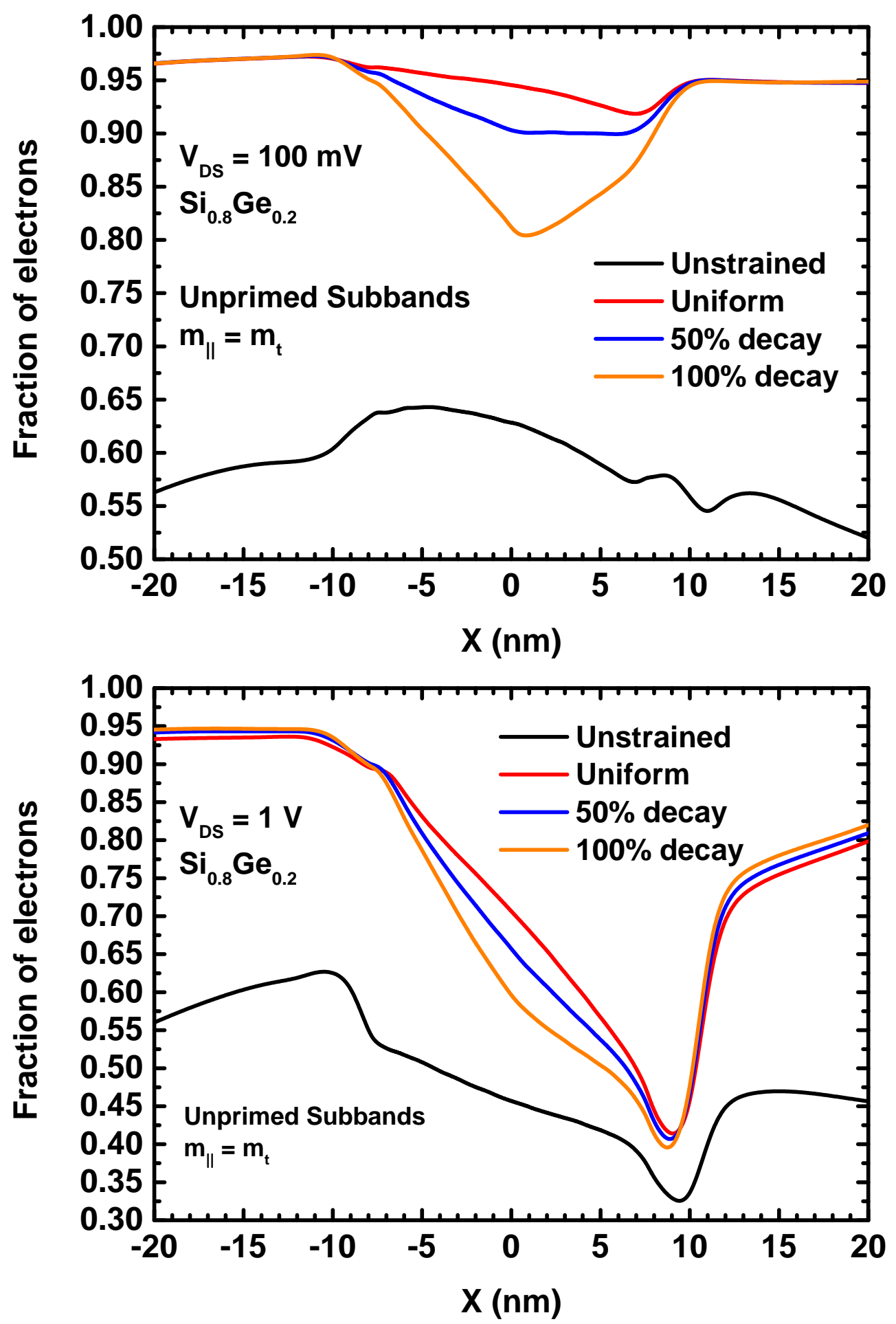

Figure 8: Population of the non-primed $\Delta_{2}$ valleys as a function of the device position for sSOI devices under low drain bias (top) and saturation (bottom) conditions. 